Accepted author manuscript (penultimate draft), 2021-03-07, forthc in Philosophical Transactions of the Royal Society B doi:10.1098/rstb. 2020.0042

Publisher's minor wording changes in published version not included here.

\title{
Digging the Channels of Inheritance: On How to Distinguish Between Cultural and Biological Inheritance
}

\author{
Maria Kronfeldner \\ Department of Philosophy, Central European University
}

\begin{abstract}
Theories of cultural evolution rest on the assumption that cultural inheritance is distinct from biological inheritance. Cultural and biological inheritance are two separate so-called channels of inheritance, two sub-systems of the sum total of developmental resources traveling in distinct ways between individual agents. This paper asks: what justifies this assumption? In reply, a philosophical account is offered that points at three related but distinct criteria that (taken together) make the distinction between cultural and biological inheritance not only precise but also justify it as real, i.e. as ontologically adequate. These three criteria are: (a) the autonomy of cultural change, (b) the near-decomposability of culture, and (c) differences in temporal order between cultural and biological inheritance.
\end{abstract}

Keywords: culture, inheritance, separateness, autonomous change, neardecomposability, stability. 


\section{INTRODUCTION: WHAT'S AT ISSUE}

Inheritance consists in the production of similarities across generations of genealogically related organisms. ${ }^{1}$ Biological inheritance is standardly understood as based on molecular processes that are part of biological reproduction (i.e. the processes, actions, or sexual interactions that lead to new organisms).

The concept of 'cultural inheritance,' as used in contemporary literature, is used in a similar but derivative manner - in order to compare the latter with the former. It thus also refers to (inter-)actions of organisms that causally contribute to the production of similarities between organisms. But these (inter)actions are independent of the biological processes involved in biological reproduction and in part also independent of the genealogical relatedness of the respective organisms. Traditionally (i.e. in the social sciences and the humanities), cultural inheritance is also known as social learning of ideas and behaviors.

The assumption that cultural inheritance and biological inheritance are separate channels of inheritance, separate transmission systems which contribute to the similarities of organisms in populations in distinct ways, is fundamental in any theory of cultural evolution. It is, therefore, more or less taken for granted and is inscribed, often implicitly, in at least three traditions that theorize cultural evolution. ${ }^{2}$ Therefore, contemporary contributions to these traditions do not focus on justifying the basic assumption at issue in this paper. The focus is rather on spelling out the different kinds of social interactions and

\footnotetext{
${ }^{1}$ For details on this concept of inheritance, see Lamm (2018). I diverge from Lamm terminologically, in two senses. First, Lamm distinguishes between "inheritance systems" and "inheritance channels," using the former for processes and the latter the way I do. The terminological choice to call the former "systems" is unconvincing since, as I will show, channels are nothing but systems. I use, as I think most authors do in the literature, 'channel' or 'system' for the divide between biological and cultural inheritance. For the diversity of concrete processes that can lead to cultural inheritance, I use the term 'transmission'. Second, Lamm assumes that inheritance is informational, while I rather follow the more nuanced account of Jablonka and Lamb (2005) which holds that some processes of inheritance might well be called informational, while others are not. Hence, you cannot put 'informational' into the definition of inheritance, as Lamm does.

2 The three often run under the following labels: dual-inheritance theory, the epidemiological account of culture, and evolutionary epistemology. They form more or less separate lineages of theorizing about cultural evolution in the second half of the $20^{\text {th }}$ century. They all take in some way or another Cavalli-Sforza and Feldman's (1981) work into account. Boyd and Richerson (1985), Durham (1991) and Richerson and Boyd (2005) can be considered as classics of dual inheritance theory. Sperber's (1996) epidemiological account of culture differs significantly in theoretical itinerary from dual inheritance theories. The third tradition, evolutionary epistemology, builds on Campbell's (1960, and later) work on 'blind variation and selective retention'. The literature on these three traditions has exploded in the last couple of years. Multiple encyclopaedia entries are now available on cultural evolution (e.g. Lewens 2018), sometimes biased towards one of the three traditions. Most of the authors of these partly separate traditions of theorizing cultural evolution involve the claim that cultural inheritance is distinct, but without a defence as detailed and multi-faceted as provided here.
} 
structures that lead to cultural variation, patterns of cultural inheritance, and eventually cultural evolution (as in many of the papers in this volume). ${ }^{3}$ In other words, that the existence of separate channels of inheritance has today the epistemological status of a fundamental assumption means that it is rarely challenged from within (meaning, in this case, the three traditions of theorizing cultural evolution). It is taken as a fundament, a starting point, used to justify or do something else, e.g. used to specify certain dynamics of cultural variation. As a fundament it is quite important since it justifies that we can speak at all about cultural change as a distinct kind of evolution. After all, one could conceptualize culture and cultural change reductively, as part of biological evolution (and some have indeed done that), rather than postulating an extra, specifically cultural evolution. Whether or not something can be reduced to something else is an important and paradigmatic philosophical issue that can have impacts on scientific practice as well. ${ }^{4}$

Thus, the question at issue in this paper is the following: is the separateness-claim just a way of looking at the world (i.e., a way of constructing theories and models that work) or does it point to something real? In other words, the question is: what justifies channelism? In reply, a philosophical account is offered that points at three related but distinct criteria that (taken together) make the separation of cultural and biological inheritance not only precise but also justify it as real, i.e. as ontologically adequate. These three criteria are: (a) the autonomy of cultural change, (b) the near-decomposability of culture, and (c) differences in temporal order between cultural and biological inheritance.

Before I can explicate these three criteria, a few further remarks are important to prevent misunderstanding or misleading expectations. First, pointing to the fact that cultural inheritance happens via processes in the brain

\footnotetext{
${ }^{3}$ When the first steps in the direction of theorizing cultural evolution in relation to biological evolution were taken, around 100 years ago, this was certainly different. Anthropologists like Kroeber (see, for instance, his 1917 paper) had to first establish, intellectually and institutionally, the study of culture as an autonomous academic field, that can function independent of biological expertise. As a result, culture and cultural inheritance emerged as field-defining explananda, i.e. the very things to be explained by cultural anthropology, justifying the independent existence of the field at a time of its emergence. See Kronfeldner (2009) for that historical episode; see Section 3(a) for what is relevant from it for this article. ${ }^{4}$ Issues about reduction can impact scientific practice, for instance, in cases in which theories of cultural evolution (as one anonymous referee put it) "do not touch upon" the separateness (because it is implicitly assumed) and rather "just make assumptions" about "cultural inheritance (without bothering about genetics) and draw their conclusions from these assumptions." After all, the ignoring of genetics derives from the implicit assumption of separateness. In short: explicit or implicit claims about non-reducibility often ground practices of ignoring. If, however, the issue of reduction is a matter of wording only, then it will very likely not make a difference for practice. I assume here, as the above-quoted anonymous referee implicitly does, that in the case at issue, the issue of reduction does make a difference in practice, a difference that distinguishes theories of cultural evolution from theories that do not treat culture as separate. An example for the latter is evolutionary psychology (e.g. as in Tooby and Cosmides 1992). See Kronfeldner (2017) on how they try to oppose theories of cultural evolution as wrongly ignoring biological differences.
} 
while biological inheritance happens via processes in gametes will not suffice for the philosophical purposes at issue, since it would still not answer why we can regard the two channels as so separate that their separateness justifies talk of a dynamically independent cultural evolution.

Second, to regard cultural evolution as separate from biological evolution does not mean that the two do not interact at the developmental and evolutionary level. Thus, this paper assumes, as most contemporary sources in the field, that only biological and cultural evolution taken together suffice to understand the evolutionary processes that we summarize under the label 'human life'. But since these interactions do not preclude the existence of cultural and biological inheritance as separate channels (and vice versa, the separateness does not exclude interaction), the two issues - justifying separation and justifying interaction - fall apart. This paper deals only with justifying separation.

Third, for those who already believe in the separateness, the paper offers a detailed and systematic justification of their belief. For those who challenge the belief in separateness (usually from outside of theories of cultural evolution), this paper offers a forceful and systematic reply. Interestingly, the latest challenges do not relate to evolutionary psychology which reduces everything to a 'long leash' of the genes, as did their predecessor, sociobiology. The latest challenges regarding channelism come from holistic approaches that unify things in a non-reductive manner. These holistic approaches challenge channelism with respect to new insights regarding epigenetic inheritance, or from the perspective of developmental systems theory. Meloni (2016: 71), for instance, writes that epigenetic inheritance is a "key mechanism in blurring the demarcation between social and biological processes," meaning cultural and biological inheritance. The most explicit attack against distinct channels of inheritance stems from Griffiths and Gray's $(1994,2001)$ turn-of-the century version of developmental systems theory. "So-called channels," Griffiths and Gray (2001: 196) claimed, are "not generally independent of one another." That this explicit attack against channelism in Griffiths and Gray $(1994,2001)$ gave way to a silent indecisiveness is telling, but it is not resolving the issue. Griffiths and Gray (2004), for instance, implicitly use some concept of channels (and actually did so already while criticizing it in 2001). They do so by referring to "extragenetic inheritance" in contrast to "genetic inheritance." Their usage of the label "extra-genetic" includes epigenetic inheritance (which is based on molecular processes that are part of biological reproduction) and cultural inheritance. It is thus not only unclear whether and how their picture allows them to distinguish between cultural and biological inheritance but - more importantly for this article - how they would justify that distinction.

Finally, this paper focuses on culture as it occurs in human beings, without denying that culture exists (in the full sense of the term) at least to certain degrees in some other animals too.

In sum: this paper presents a novel and systematic account of how to 'dig' the channels of inheritance, that is: how to understand them in a precise and in-depth manner. To the best of my knowledge such an account is not existing 
at the level of philosophical abstraction and precision envisioned here and thus fills a gap in the literature on foundations of theories of cultural evolution. ${ }^{5}$

The to-be-defended core claim is the following: even though there is indeed a high degree of dependency between epigenetic transmission in relation to genetic transmission (after all, both belong to the set of molecular processes that explain biological reproduction), the dependency between the cultural channel in relation to the biological channel is vanishing in comparison to that. This is so, as I will show, since there are related but distinct dynamic independencies between cultural and biological inheritance that are crucial to understand the specifics of culture and cultural change. These are: (a) Cultures can (and often do) take-off, i.e. change without a concomitant populational change in biologically inherited developmental resources, (b) cultural inheritance is near-decomposable from biological inheritance, and (c) it lacks a temporal order because of the importance of choices made by the agents involved.

\section{MAIN EXISTING ACCOUNTS OF DEFENDING DIFFERENT CHANNELS AND MODES OF INHERITANCE}

The account defended here builds on allies, i.e. researchers who have already made some important steps into the terrain by delineating different kinds of transmissions within the respective channels. Many in the field will immediately think of the tripartite grid that allows one to distinguish between vertical, oblique and horizontal transmission modes of cultural inheritance. The vertical transmission mode runs from parents to offspring, while the oblique mode operates between genealogically unrelated individuals of different generations. The horizontal mode limits the latter to individuals of the same generation. This tripartite grid goes back to Cavalli-Sforza and Feldman (1981), the locus classicus of contemporary debates on gene-culture co-evolution.

The common-sense view takes biological inheritance to be simply vertical. Hence, can't we establish the difference between biological and cultural inheritance by pointing to the fact that culture is vertical and horizontal and oblique, i.e. not only vertical? Or by pointing to the fact that culture can change quicker than nature? ${ }^{6}$ Indeed, that is the basic idea usually at play and it will be utilized in this article too. Yet, the devil is (as always) in the details. Using the three modes to differentiate between cultural and biological inheritance is less straightforward than it seems at first glance since both cultural inheritance and biological inheritance can be vertical, oblique and horizontal, and both can be quick and slow. That is why the question arises: what, in fact, justifies regarding the two channels as distinct? I aim to show that

\footnotetext{
${ }^{5}$ What follows is already part of Kronfeldner (2018), although in a form dispersed across the Chapters of that book.

${ }^{6}$ See Lewens (2015: 91) who simply points at the difference between "swift" horizontal cultural inheritance and "relatively slow" biological inheritance to save "channel talk".
} 
it matters why a transmission mode is operative, whether the mode can be switched, and which proportions regarding the operative modes result from that. Looking at these details will be part of the solution to the question at issue in this paper. Before I can go there, two other accounts of distinguishing channels and transmission modes of inheritance need to be mentioned. They are the most detailed and most influential accounts available as of today, given the topic of this article, and are singled out because of that.

Jablonka and Lamb (2005) distinguish between two qualitatively different kinds of transmission within the cultural channel (symbolic and behavioral transmission), and two analogous kinds within the biological channel (genetic and epigenetic transmission). For Jablonka and Lamb, symbolic cultural transmission and genetic transmission stand out since they involve, according to them, informational replicators, whereas behavioral cultural transmission and epigenetic transmission do not. Irrespective of whether that commitment to information and replication works out or not (which is irrelevant for the topic of this paper), their account does unfortunately not offer a detailed justification of the more basic divide between cultural and biological inheritance. They simply employ it in order to introduce the further distinctions involved in their account. The account presented here is thus meant as a complement to their model, but one that is neutral with respect to their claims about the special features of informational transmission.

Equally consistent with the account developed here is Odling-Smee's (2007, 2010) theory of niche inheritance. He distinguishes biological inheritance from niche inheritance by characterizing the first as direct transmission happening internal to the organism and the second as indirect transmission happening outside the respective organisms. The account I present in the following is not in opposition to his account since I take his account to entail that cultural inheritance is a case of external-to-the-body niche inheritance. It is distinct from pure ecological inheritance, which does not involve any social learning, but at the same time a crucial component of the broader category 'niche inheritance' since it circumscribes "the primary means by which humans engage in the universal process of niche construction," as Odling-Smee and Laland (2011: 220) write. Interestingly, Odling-Smee's account assumes the body boundary as decisive for delineating direct-internal and indirect-external channels of inheritance. The account provided here is compatible with that but goes deeper: it does not assume the body boundary as a given; it rather provides a philosophical foundation for the used demarcation line between direct-internal biological inheritance (molecular, happening via processes in the gametes) and indirect-external cultural inheritance (ideational or behavioral, happening in the brain) by justifying the ontological internal-external dividingline dynamically. That dynamic picture takes the body boundary to be resulting from (b) near-decomposability. After all, what is philosophically decisive is not that the line is drawn at the body boundary but why that is justified. ${ }^{7}$

\footnotetext{
7 Regarding the question whether cultural and ecological inheritance are so entangled that they cannot be separated, I will stay neutral. It is an issue that is as difficult as distinguishing
} 
In sum, three existing accounts (Cavalli-Sforza and Feldman, Jablonka and Lamb, Odling-Smee and Laland) are in the background of what follows. Yet, they do not suffice to defend channelism against the challenges mentioned in Section 1. What is needed is an explicit and in-depth depiction of the independence(s) of cultural inheritance in relation to biological inheritance.

\section{A TRIPARTITE DEFENSE OF CHANNELISM: AUTONOMOUS CHANGE, NEAR-DECOMPOSABILITY, AND DIFFERENCES IN TEMPORAL ORDER}

\section{(a) The take-off autonomy of cultural inheritance}

The first justification I offer for regarding cultural and biological inheritance as independent lies in the frequently mentioned autonomous change of culture. Even though culture can be very conservative (changing slowly), it can (if needed) change very quickly. If it does so, culture is like a fast track of evolution and changes without a concomitant change in the biologically inherited resources of the respective population. Change introduces variation in time, and over time that variation is visible also in space. Hence, the fact that culture can change without a concomitant change in biologically inherited resources explains why - over evolutionary and developmental time - culturally inherited developmental resources may vary in space and time (and often do so) even if the relevant biologically inherited developmental resources do not. 8

It follows that culture can take off and is in that sense 'superorganic': autonomously changing and varying. How that looks like over time, has been nicely depicted in a graph from Alfred L. Kroeber's famous (1917) paper The Superorganic (see Figure 1).

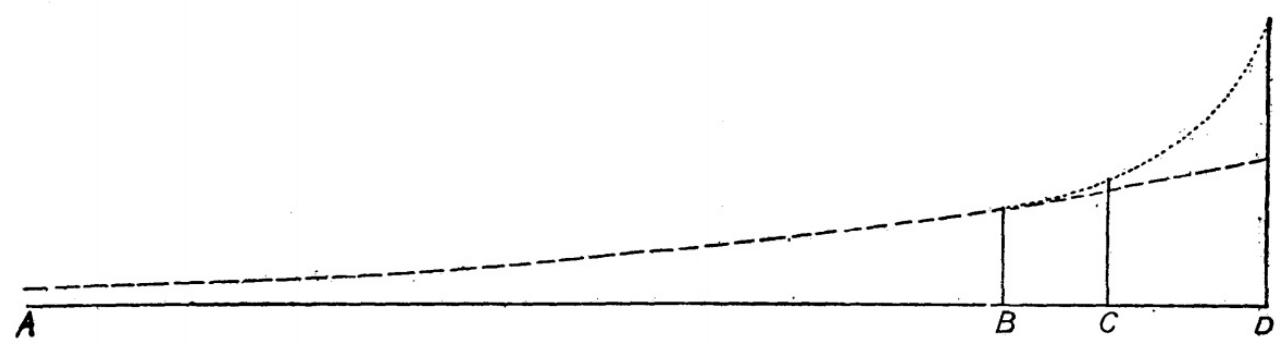

Figure 1. Culture taking off. The horizontal axis represents time and physical persistence, which is taken to not evolve at all. The vertical axis represents accumulation of changes (or increase in complexity even). Cultural evolution (the dotted line) is autonomously changing in relation to biological evolution (dashed line). $\mathrm{B}$ is the first animal using culture, while $\mathrm{C}$ denotes the beginning of our species and $\mathrm{D}$ the end of the

between biological and cultural inheritance. It might well be that all three independencies utilized here would help for that question too, but whether they do or not is irrelevant for the claims made in this paper. See Odling-Smee and Laland (2011) for their take on the relationship between niche inheritance and culture.

${ }^{8}$ Note that culturally inherited resources can be as universally distributed as biologically inherited ones, and vice versa: biologically inherited resources can be as local as cultural resources, i.e. differentially distributed in time and space, without there being 'races' or essentialized cultures. The differential distribution of the biological foundations for lactose tolerance (a world-map of shadings of different frequencies, without clear borders) is a case in point and an example that will be utilized below for further points. 
nineteenth century. (Reprinted from Alfred L. Kroeber, “The Superorganic.” American Anthropologist 19, no. 2, 1917: 163-213, 211. Public domain.)

As Kronfeldner (2009) explained, Kroeber's account is built on an antiLamarckian concept of inheritance which allows decoupling biological and cultural inheritance. ${ }^{9}$ After all, for a Lamarckian, who believes in the inheritance of acquired characteristics, biology ("inherited" in the Lamarckian dictum) and culture ("acquired" in that same dictum) always change together (small fluctuations ignored). For non-Lamarckians like Weismann and Kroeber, it was of utmost importance at the beginning of the $20^{\text {th }}$ century to explicitly stress that culturally transmitted developmental resources can change (within and across generations, at the individual and at the populational level) without any concomitant change in those developmental resources that are transmitted via biological reproduction. This independence of change is the foundation for the take-off Kroeber so nicely depicted. Thus, the history of Neo-Darwinism, which opposes Lamarckian inheritance (which Darwin did not), is the history of channelism and with that it is the history of theories of cultural evolution. You can certainly find the seeds for that idea already in Darwin himself (e.g. when he refers to language evolution). Yet, only in Weismann and Kroeber, both attacking Herbert Spencer's monist version of thinking about evolution in and outside of biology, can we find a detailed defense of an autonomously changing cultural channel of inheritance. ${ }^{10}$

In part, the take-off autonomy certainly relies on the fact that culture is often functioning in a horizontal mode of inheritance. It can easily change quickly since it can spread independent of biological generations. It does not have to wait for a new generation of organisms. This will be taken up below again.

Defending the take-off autonomy of culture, Dobzhansky (1956: 119) concluded that one cannot predict the course of culture from knowledge of biological developmental resources alone, "no matter how complete" that knowledge is. I will below add that it can also not be predicted from knowledge about the cultural channel existing as a separate one. Viewed from the outside, culture means caprice and that is its power too.

Griffiths and Gray (2001, as quoted above) simply seem to ignore the take-off autonomy of culture, even though it is compatible with the interactionist consensus they rely on (i.e. that nature and culture interact at the level of development and at the level of evolution). For instance, autonomous changes of culture do not prevent that there are feedbacks between cultural evolution and biological evolution. Such feedbacks have been defended as part of gene-culture co-evolution theories, e.g. with respect to the evolution of lactose tolerance, probably the most paradigmatic example for gene-culture co-

\footnotetext{
${ }^{9}$ See Kronfeldner (2007) for claiming that cultural evolution is not (in any interesting sense) Lamarckian.

${ }^{10}$ For details and references regarding Weismann and Spencer, see Kronfeldner (2009).
} 
evolution. ${ }^{11}$ The story behind this example is the following: in some areas of Eurasia, people heavily relied on dairy farming, which spread first in the Levant then in Europe. When favorable mutations occurred (mainly the $13910^{*}$ T allele, given current evidence), some people became able to digest cow milk even as adults (which they weren't till then). This, in turn, fostered dairy farming, which fostered the selection of the respective mutations for lactose digestion, and so on. This is coevolution, biology and culture interacting ontogenetically as well as phylogenetically, even though there are no genes for dairy farming and no Lamarckian inheritance, maybe not even epigenetic inheritance.

Explanations of the evolution of lactose tolerance that include cultural inheritance (as a factor that determines the fitness landscape of the genetic factors involved) have been shown to better fit the available data than alternatives (e.g. the so-called calcium uptake hypothesis). In addition, given current state of the art, cultural inheritance of dairy farming practices took the lead in the co-evolutionary process. Evidence for that comes, for instance, from the pottery used for cheese making, which is now archaeologically studied to track the respective co-evolution. This means that for some time culture (dairy farming practices) changed without a concomitant change in biologically inherited developmental resources. Later, biology (the respective mutations) followed and the two spread more or less unisono, in harmonious gene-culture interaction, leading to co-evolution, each nudging the other on, but each still marching on its own, in its distinctive manners.

To conclude: as long as culture can change independently from biologically inherited resources or can take the lead in co-evolutionary processes, cultural and biological inheritance are decoupled and make up two separate, evolutionarily relevant channels of inheritance. After all, autonomous change presupposes separateness, as Gray and Griffiths (2001, as quoted above) noted too, even though they did so in order to deny the separateness and independence of the channels.

\section{(b) Near-decomposability}

The discussed take-off autonomy is related to a second kind of independence, namely near-decomposability, which allows to establish the boundaries of the two different channels of inheritance as real but based on dynamic features, i.e. at the level of causal interactions. Merlin (2010: 209) provides the core clue: if there are specific channels of inheritance, then the "interactions among their respective own elements" should be "stronger than the interactions among elements belonging to different subsystems" (i.e. channels) of the overall inherited developmental system. Since Simon (1962), such cases of moreinteraction-within-than-between subsystems of a whole are called cases of "neardecomposability". The qualifier "near" is important. Full decomposition of a

11 Aoki (1986) and Feldman and Cavalli-Sforza (1989) are seminal references for the case, developing the first models for it. See Gerbault et al. (2011) for an update on the case. Compare Lewens (2015: 86-93) who also refers to it as a paradigmatic case to understand how to refer to the channels of inheritance. Research on the case is still ongoing. 
system would mean that the system actually has fallen apart. If, on the other hand, interaction within a discernable system is equally distributed, then the system is a whole but not decomposable. The ontological delineation of parts (sub-systems) becomes difficult if not impossible in such a case. It follows that only if there is near-decomposability in a system (some but not complete decomposability) can one safely draw ontological boundaries around parts of a bigger whole. And this is what helps for the case at issue here too. What needs to be shown is that there are near-decomposable channels (subsystems) of inheritance.

Take the case of lactose tolerance again. How does the principle of neardecomposability apply to the case? The dairy farmers in the Levant who changed - through their cultural practices - the prospects for the selection of the genetic change that made lactose tolerance in adults possible, had quite some daily interactions with each other: they raised the cattle, produced milk, produced and used the pottery that allowed them to store the milk and make cheese (which they were able to digest easier since it contains much less lactose); they also guarded the cheese from being stolen or getting rotten, and so on. Most of these interactions involved social learning mechanisms. At the same time, it is very unlikely that genetic factors (which made lactose tolerance biologically possible or impossible) made a causal difference for that daily business of dairy farming (from all we know so far). In addition, new styles (e.g. of how to make pottery to improve cheese making) might have spread quicker than the mutations that might have been around already. That means: people socially learned and enacted the practices connected to dairy farming thanks to a complex and high-frequency network of social interactions, while the mutations that showed up were initially not making any salient difference to these daily social interactions. And vice versa: it can also be assumed that the cultural practices had no direct developmental effect on the mutations themselves. This holds even though, to the best of my knowledge, it cannot yet be excluded that there were some epigenetic effects and even though on the evolutionary timescale (i.e. in the long run) the cultural practices made a difference for the spread of the mutation, which is precisely the point of the coevolutionary account. Finally, there might have been some developmental feedbacks and influences (e.g. via epigenetics), but compared to culture interacting with culture and genes interacting with genes, these cross-channel interactions were very likely negligible. It is thus safe to claim that the two channels have been near-decomposable, at least in the case at issue. Given all the above, it can be concluded that the principle of near-decomposability applies to the case at issue.

Since the case is a paradigmatic case, one can extrapolate to other cases of co-evolution. It is thus justified to draw an ontological boundary between a biological and a cultural channel of inheritance whenever it can be assumed that there are more causal interactions within the system of learning and within the system of biological inheritance than between the two.

A channel of inheritance, given the view defended here, is thus not a material entity. It is not a physical barrier preventing equally distributed 
interaction of the elements, like a wall or tube, although it can certainly involve such physical barriers. A channel is a real pattern of causal interactions, a pattern of differential distribution of causal interactions that allows drawing a morewithin-than-between causal boundary between parts of a bigger whole. ${ }^{12}$ In the case at issue, it justifies drawing an ontological boundary where we usually draw it: at the body boundary. From that it follows that one channel of inheritance is internal to the bodies and based on molecular processes involved in biological reproduction, while the other is more 'ephemeral': external to the bodies, happening between people, known since ages as: culture based on the social learning of ideas and behaviors.

To conclude: as long as it can safely be assumed that neardecomposability applies, the distinction between cultural and biological inheritance is ontologically adequate - that is, cutting reality at one of its important joints.

\section{(c) Differences in stability-granting temporal order}

Over an evolutionary timescale, the two channels also differ in a third sense, a sense that stems from the more or less autonomous choices of the individual agents involved; a sense that leads to differences in temporal order, which amounts to differences in stability and in part explains differences in actual transmission dynamics. The term 'temporal order' refers to structural features that relate to the modes of inheritance (in Cavalli-Sforza' and Feldman's sense) which lead to differences regarding the stability that the channels provide for inheritance, i.e. the probability of long-term re-occurrence of the respective developmental resources across generations. It is an 'order' since it refers to structural features; it is 'temporal' since it influences dynamics over time.

The to-be-defended claim is the following: the biological channel conveys - on average - much more stability than the cultural channel since the resources traveling within it normally cannot switch its vertical mode of inheritance, while in culture, individual agents decide which mode to use and do so based on the content of the to-be-inherited item at issue. This freedom to choose leads to the absence of a characteristic temporal order, which leads to less stability of the channel (compared to the biological channel), which explains (in part) the high degree of unpredictability of actual cultural transmission dynamics.

As mentioned, vertical transmission runs from parents to offspring, while oblique and horizontal transmission is between genealogically unrelated individuals. Cavalli-Sforza (2000: 179-187) later refined this tripartite taxonomy

\footnotetext{
${ }^{12}$ Another example that illustrates this point is the existence of different departments in universities. One cannot just 'see' that universities have different departments by looking at physical entities (e.g. the buildings, the walls). One needs to 'look' at the people, i.e. their causal interactions, to discern parts of the whole. Only if the elements (members of the universities such as professors, staff, and students) interact more within individual departments than between different departments is it justified to conclude that there are in fact different departments of that university.
} 
into a more fine-grained grid of four modes: a vertical mode plus three different kinds of horizontal modes of transmission, the latter distinguished according to the number of senders and receivers involved. If applied to cultural transmission, the following three horizontal modes are the result. First, there is a one-to-one communication mode, as in peer-to-peer social learning. Second, a magistral transmission mode, by contrast, is a one-to-many communication pattern, as in mass media or cases where an authority enforces a cultural item on a population via teaching, by decree, political pressure, or simply by network power (see Noebel et al., this volume). There is also, third, a concerted transmission mode which involves a many-to-one communication order, where a group of people exerts social pressure on a new member, as in religious cults and perhaps social media. All modes of cultural inheritance can be further divided into transmission processes that exhibit some bias (such as "copy-thebest" or "copy-the-neighbor," in which learning is biased towards certain senders, e.g. the best in the group, the richest in the group, or the closest neighbors).

All these modes differ in terms of structure, i.e. the kind of change (how a new item in the population spreads). The actual dynamic (i.e. how quickly things change) depends on contextual factors and on four core interacting factors: the mentioned structural features, the rates of novelty occurring, the fidelity of transmission (frequency of copy-errors), and (last but not least) selective pressures.

Vertical transmission is usually rather stable, i.e. slow and reliable and is so as a result of its temporal order (one-to-one, bound to biological generations), its low rate of novelty being introduced, and a relatively high fidelity of transmission. Yet, vertical transmission is less reliable than concerted and magistral transmission since the latter often have a still higher fidelity of transmission. Religions, for instance, usually travel a vertical or concerted transmission mode and show because of that a high stability over time. Magistral transmission, by contrast, is very handy for quick spread of novelty with a quite high fidelity, which is why it is used in schools and by governments. Yet it is usually less creative and can be still slower compared to peer-to-peer learning which has a comparatively low fidelity, a high rate of novelty, as fashion (dress codes) or any game of telephone illustrates. ${ }^{13}$

As mentioned, the actual speed of change (evolution), depends on many factors, the four core ones mentioned above and contextual factors. As a result, actual transmission dynamics for concrete cases of evolution are quite complex. ${ }^{14}$ The following aims to show that the specificities of cultural

\footnotetext{
13 The speediness of horizontal transmission is also why descriptions of some cultural items as 'spreading like viruses' are so tempting, especially after 2020, when the world had to witness so forcefully that the horizontal transmission of the Covid-19 virus, leading to a history-making pandemic, so easily outperforms government decrees spreading in a too conservative magistral mode.

${ }^{14}$ For a book-length treatment of these complexities, see Morin (2015). The book is not oriented at distinguishing channels but at understanding, as the title says, "How traditions live and die."
} 
inheritance crucially (but not exclusively) derive from one peculiar and unfortunately often forgotten factor: the choices of individual agents.

The argument rests on a claim made by Claidière and André (2012). They criticize the assumption that the temporal order and actual transmission dynamics in cultural inheritance are a result of different modes of transmission. They argue that it is just the other way round: the transmission modes (and thus the temporal order) are the result of transmission processes that are determined not by different modes (i.e. structural features) but by the content of what is transmitted and by the beliefs and preferences of the individual agents transmitting culture and thereby selecting cultural items. The beliefs and preferences operative in the choice (of sender and receiver) include beliefs and preferences about when to communicate or learn something and to or from whom.

A core argument they use for this claim (and that is the decisive point for this paper as well) is that a cultural trait can change its mode of transmission from vertical to horizontal. Imagine the following: The availability of water changes, and you learned from your parents how to prepare potatoes by cooking them in water, and that is all you think one can do to potatoes to use them as food. Given the new evolutionary affordances, your friends, who in contrast to your parents quickly adapt to the new evolutionary affordance, successfully try to bake the potatoes in the oven, without using any water. You learn this new behavioral 'technology' from them. The actual content of the cultural item transmitted (ideas about how to prepare potatoes) and the evolutionary affordances (whether adaptation is quickly needed or not) determine whether it makes sense to do as your friends do or not. Thus the mode in which ideas and behaviors about how to prepare potatoes travel can change to fast track (horizontal transmission), leaving the 'conservative' security track (vertical transmission) behind.

By contrast, biologically inherited resources normally cannot change their mode of transmission just because their 'content' in relation to evolutionary affordances would make that beneficial. ${ }^{15}$ Mitochondrial DNA is transmitted maternally, whatever it does in its context; nuclear DNA is (in sexually reproducing species) transmitted biparentally; methylation patterns are transmitted epigenetically; and other cellular resources are transmitted at the cellular level, etc.

The mode of transmission in cases of biological inheritance is independent of the 'content' of the item being transmitted and thus is also independent of evolutionary affordances, at least for a very long time (since certainly the way these molecules are transmitted ultimately evolved as well and will further evolve in relation to evolutionary affordances). The mode of transmission in biological inheritance depends on the kind of molecule transmitted. It is mechanically fixed by the material constitution and the mechanisms of biochemical reactions that make inheritance possible for such a

15 "Content" in the biological case is here interpreted functionally, as "what the resource does for the organism." 
kind of molecule. ${ }^{16}$ Finally, most of the mechanisms involved in biological inheritance are vertical, with the exception of lateral gene transfer, which rarely occurs for multicellular organisms (on current evidence).

What follows from this for the task of delineating biological and cultural inheritance? Claidière and André (2012) have used the above to question the usefulness of distinguishing between different modes of inheritance within the channel of cultural inheritance. Yet, their argument can also be used to strengthen the divide between cultural and biological inheritance, namely as operating in distinct ways. If Claidière and André are right, and I believe they are, then there are two quite distinctive ways in which developmental resources can be transmitted over time: in a mostly content-independent manner (biology) and in a content-dependent manner (culture).

On average, the difference between content-dependent and contentindependent transmission has important consequences for what I above called stability that the respective channel of inheritance promises. As indicated, a channel conveys stability if change is comparatively slow and transmission reliable. The claim that I want to put forward is this: the biological channel has a high-level of stability, determined by the structure of the molecules and the mechanisms of transmission, whereas cultural inheritance has no such predetermined stability.

- $\quad$ The biological channel leads on average to much more stability since the resources traveling within it normally cannot switch their mode of inheritance and thus stay predominantly vertical, a mode that has a high fidelity and is characterized by slow change and thus can contribute high stability. This holds even though epigenetic inheritance does not exhibit high stability and functions more as a quick but reliable finetuning mechanism within the biological channel, and even though there is some lateral (i.e. horizontal or oblique) gene transfer. The decisive point for the overall channel is the proportions and the vertical mode simply dominates 'the show'.

- Culture, by contrast, is normally so dependent on the choices of agents who react to the contents of culture and the respective contextdependent affordances that - on average - it is much freer, less predictable and less secure in terms of stability over time, even where fidelity (no copying mistakes made) is high.

\footnotetext{
${ }^{16}$ Viruses are a notable exception for this claim about 'fixed' modes of biological inheritance. As Isabella Sarto-Jackson (personal communication) remarked, with reference to Pellett et al (2012): sometimes genetic factors can indeed be regarded as switching modes of inheritance. For instance, herpes viruses can be transmitted vertically (by biological reproduction, from a parent to descendants) as well as horizontally to another adult, and even at the same time. But note that in order to really be analogous to the cultural case, the switch needs to happen in response to new evolutionary affordances, and it needs to be reversible to count as equally contentdependent. It goes beyond the scope of this paper to determine whether that applies to the case. An anonymous referee has pointed to the fact that for viruses it can indeed happen in a responsive and reversible manner, giving further credence to a by now standard comparison of ideas and viruses.
} 
Viewed from the outside, culture is pure caprice: it can have high stability and it cannot. The channel itself does not allow for any predictions of the probability of reoccurrence of the developmental resources that travel through this channel. Hence predicting actual cultural transmission dynamics is much harder than predicting transmission dynamics in biological inheritance.

The freedom of choice about what to transmit (send) and what to learn (receive) leads to a pattern that looks like caprice if viewed from outside. That caprice is built into cultural inheritance, at least for humans and other animals, and it is the crucial difference between cultural and biological evolution. That crucial difference also goes, traditionally, by the name of 'freedom' and grounds (a) the take-off autonomy, as well as (b) the near-decomposability, and (c) the peculiar absence of a temporal order in cultural inheritance, since it provides the splendid flexible stability that makes a full-blown cumulative culture such a powerful evolutionary force, so powerful that many believe that it 'made us human'. I will say more in Section 4 on the relationship between choice and (a)-(c); before I go there, let me illustrate the 'caprice' I mean a bit more.

Danchin et al. (2011) distinguish between genetic, epigenetic, parental, ecological, and cultural transmission. They graphically characterize the temporal order of the different channels that they distinguish by pointing to vertical versus non-vertical transmission modes (Figure 2).

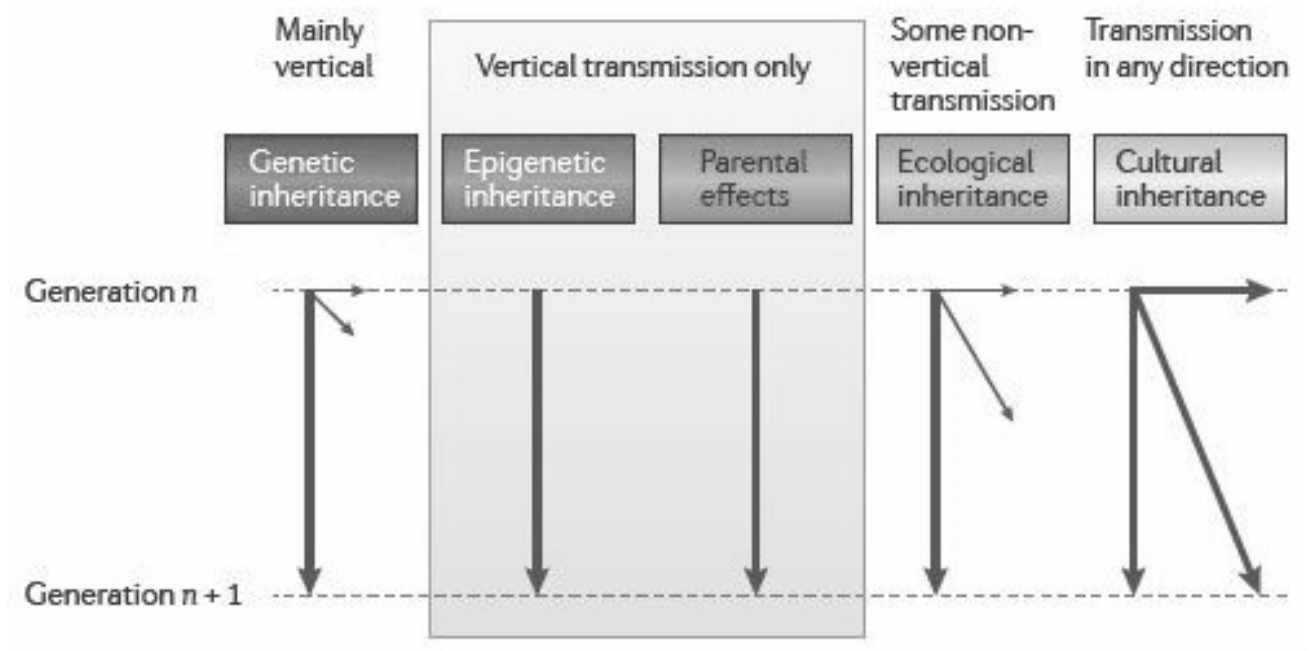

Figure 2. Channels of transmission. The channels of inheritance are characterized as vertical, horizontal and oblique transmission modes, represented as arrows pointing vertically, obliquely to the right, and horizontally to the right. (Reprinted with permission from Springer Nature: Nature Review Genetics; Danchin, Étienne, Anne Charmantier, Frances A. Champagne, Alex Mesoudi, Benoit Pujol, and Simon Blanchet. 2011. "Beyond DNA: Integrating Inclusive Inheritance into an Extended Theory of Evolution." Nature Reviews Genetics 12 (7): 475-86, p. 481), Copyright (): Macmillan Publishers Limited (2011).

Even given lateral gene transfer (the two small arrows in the field of genetic inheritance in Figure 2), the figure illustrates that cultural inheritance is quite peculiar in terms of its temporal order since simply no transmission mode 
dominates. ${ }^{17}$ Above, I described the fact that 'no mode dominates' as the absence of temporal order. One can certainly also interpret it as a special kind of order, as anarchism can be counted as a special kind of political order, despite the absence of hierarchies. Whether it is interpreted as absence of order or as a quite unusual kind of order does not matter much since it does not impact the substantial issues involved. Hence, I prefer to stay neutral with respect to that interpretative issue, even though I utilized the language of 'absence'. What is important is rather how to explain what I call 'absence of temporal order' or 'caprice'.

With Claidière and André (2012), one can explain it very aptly. In cultural inheritance, no mode dominates since transmission is contentdependent. Mode of transmission thus can change at any time, not just from one cultural domain to another (e.g. from religion to fashion) but also for a particular cultural trait (e.g. how potatoes are prepared; how milk is used). The actual transmission dynamic, in the end, depends on lots of contextual variables, since these influence the agent in the choice that determines whether transmission will be vertical, horizontal, or oblique, whether novelty comes easily, and also whether transmission is characterized by high fidelity.

The fact that biologically inherited resources are bound to a mostly vertical mode of transmission is one of the reasons why the biological channel is regarded as characterized by, on average, higher stability. Once genetic factors (e.g. the $13910^{\star} \mathrm{T}$ allele that explains lactose tolerance in European populations) are in a genetic lineage, there is nothing in the 'mechanics' of the channel that prevents them from staying there, virtually forever. (That there can be mutations and that they can be selected against is another matter.) The addition 'on average' is certainly important since epigenetic inheritance is rather unstable and there is lateral gene transfer. The important difference is, as mentioned, one of proportions. In biological inheritance (at least for animals) the vertical mode dominates and in culture it does not - because of the freedom of the agents to choose. ${ }^{18}$

\footnotetext{
17 The figure has one glitch: it classifies parental effects as cases of inheritance. Although parental effects influence gene expression and although they can be inherited, they are not qua being parental a case of inheritance, as Merlin (2017) argues. What parental effects qua parental effects are doing is introducing novelty into the next generation's somatic line. This novelty can be adaptive but it does not have to be, and it can be epigenetically inherited to the still next generation but it does not have to.

${ }_{18}$ This helps to answer a worry that Buskell (2019) raised with respect to the claims defended here, and first stated in Kronfeldner (2018). He pointed to Heyes (2018) showing that there are important cases of culture being very stable and thus evolutionarily very important. That is certainly true (and important), but in and of itself it does not change the proportions of modes operative in cultural inheritance. In other words, pointing to such cases is not sufficient to show that, overall, cultural inheritance is as stable as biological inheritance or that cultural inheritance, overall, is dominated (as biological inheritance) by a vertical mode. Having said that, it needs to be mentioned that Buskell (2019) also raised the issue of whether this difference in temporal order grounds differences in 'explanatory importance' of the two channels as discussed in Kronfeldner (2018). Since explanatory importance depends on the explanandum at issue and the kind of explanation targeted thereby, a reply to that issue goes beyond the scope
} 
With this it becomes explainable why culture can take-off and even why it has evolved: because it can change quickly if necessary. Certainly, as mentioned, culture can also be quite conservative (e.g. if it is vertical) but, as discussed above, it is at the same time unique in being nonetheless the perfect fast track of evolution. Jablonka and Lamb (2005: 298) therefore speak of culture as being in "the driver's seat." That is even the point of culture: it is the function of the genetic mode in the evolution of multicellular organisms to have high stability: biology "keeps the books", while culture is in "the driver's seat". The genetic mode does not allow for a similar fast-track mode and the resulting flexible but potentially stable changes everyone knows and admires from culture.

The decisive point is, as mentioned, that what cultural transmission looks like depends a lot on the content and the context (and that includes the choices of the individuals involved). The channel allows for all kinds of dynamics since it is flexible and stable. The trajectory of culture is thus, to repeat, not predictable from the channel itself; the trajectory of biology is, by contrast, much easier to predict (compared to culture at least).

To conclude: If there are processes that differ so much in temporal order as do biological and cultural inheritance, then they should be regarded as separate and independent in that sense.

\section{THE RELATIONSHIPS BETWEEN THE THREE INDEPENDENCIES}

Since the lack of temporal order in culture is to a great extent, if not completely, derivable from the importance of choices of individual agents, and since quick changes will often be based on non-vertical modes of inheritance, the lack of temporal order grounds and facilitates (a) the take-off autonomy, as well as (b) near-decomposability. In other words, both (a) the take-off autonomy and (b) near-decomposability stem from culture being able to change more quickly (if necessary) by going horizontal or oblique, which equals (c) the absence of a preset temporal order. If necessary, we choose to diverge from what we learned from parents (neither drinking milk, nor making cheese) and adopt new habits (making cheese; drinking milk) if we think these new habits pay off, i.e. help to adapt quickly to new challenges or opportunities.

Thus, at the core of all three independencies lies our good old 'freedom': the ability to introduce new ideas or behaviors (independent of a concomitant novelty in biologically transmitted developmental resources) and the ability to choose - what to communicate and learn, when and to or from whom. The ability to innovate and to choose as one thinks right are the cognitive mechanisms that evolved to allow for the 'events' (i.e. the causal interactions) that make up culture and make it so special.

It is important to note that (a), (b) and (c) are nonetheless distinct. Even though (a) take-off autonomy requires quite some (b) decomposability so that

of this paper and thus has to wait for another occasion. Yet, I wanted to take the opportunity to say: point taken. 
the parts can develop independently, there can be (b) near-decomposability without any long-term (a) take-off happening, as in the case of lactose tolerance, in which nature and culture changed in a dependent but near-decomposable manner. Also, (a) and (b) could exist even if culture were fully bound, let's imagine, to a horizontal mode only (and the choices characteristic of it). Imagine a case of a biological life form, where biological generations (and the family bonding and social hierarchies that characterize vertical or oblique transmission) do not exist. In addition, imagine that magistral and concerted transmission are somehow cognitively disabled, so that there is horizontal oneto-one transmission only. As long as choices are made (about when and what to learn from whom) and independent of any respective 'genetic' determinants, (a) and (b) can still occur, even though there is, in this fictional life form, (c) a fixed temporal order, namely the one resulting from horizontal transmission as the one and only available mode of cultural inheritance.

\section{SUMMARY}

This paper has provided three reasons for regarding cultural and biological inheritance (as they occur in human life) as separate channels of inheritance. The argument from take-off autonomy states that the cultural inheritance channel is decoupled from biological inheritance, i.e. the transmission and thus population-level distribution of cultural resources can change and vary without a concomitant change or variation in biologically transmitted developmental resources. The argument from near-decomposability shows that biological reproduction and learning build empirically discernible channels of inheritance. Distinguishing channels is thus ontologically adequate. The argument from temporal order states that - even though both the biological and the cultural channel can be vertical, oblique and horizontal - cultural transmission is content-dependent and because of that quite peculiar - capricious - in terms of the stability it conveys since there is a lack of temporal order (no mode dominates). As part of that, the power of choice emerged as the foundation for all three independencies: take-off autonomy, near-decomposability, and the lack of temporal order. 


\section{ACKNOWLEDGEMENTS}

This paper has previously been presented at various events, even though in a more rudiment form. I want to thank the organizers and audiences of the colloquia of the ERC Research Group "The Emergence of Relativism" (University of Vienna), the "Human Mind Project" Group (University of London), and the Konrad Lorenz Institute for Evolution and Cognition Research (Klosterneuburg). The same holds for the organizers and audiences of the OSCAR Workshop at Central European University (Budapest), the Workshop on "Genographies" at the Center for Interdisciplinary Research (Bielefeld University), the Lecture Series on "Biology of Genomes" as part of the Center of Advanced Studies (Ludwig-Maximilians-University Munich), the "Kult-Ur-Mensch" Conference of the Research Group “The Role of Culture in Early Expansions of Humans" (Heidelberg Academy of Science), and the 2019 Conference of the German Society for Philosophy of Science (Cologne). I also want to thank: the editors of this issue, two anonymous referees, Mathieu Charbonneau, Alexander Reutlinger, Isabella Sarto-Jackson and Carola Stotz for their helpful remarks on earlier versions; MIT Press for allowing me to doubleuse the ideas and material used in my 2018 book "What's Left of Human Nature"; and László Kőszeghy for permission acquisition and copy-editing. Open access of this article has kindly been made possible by funding from Central European University's Research Excellence Fund.

The author has no conflict of interests to declare. 


\section{REFERENCES}

Aoki, K. (1986). A stochastic model of gene-culture coevolution suggested by the "culture historical hypothesis" for the evolution of adult lactose absorption in humans. Proceedings of the National Academy of Sciences, 83, 2929-2933.

Buskell, A. (2019). Review of "What's Left of Human Nature? A Post-Essentialist, Pluralist, and Interactive Account of a Contested Concept." International Studies in the Philosophy of Science 32, 2: 137-40.

Boyd, R. and Richerson, P. J. (1985) Culture and the Evolutionary Process. Chicago, IL, University of Chicago Press.

Campbell, D. T. (1960). Blind variation and selective retention in creative thought as in other knowledge processes. In G. Radnitzky and W.W. Bartley (Eds.)

Evolutionary Epistemology: Rationality and the Sociology of Knowledge, 91-114. Chicago, IL: Open Court.

Cavalli-Sforza, L. L. (2000). Genes, Peoples and Languages. New York: North Point Press.

Cavalli-Sforza, L. L., \& Feldman, M. (1981). Cultural Transmission and Evolution: A Quantitative Approach. Princeton, NJ: Princeton University Press.

Claidière, N., \& André, J.-B. (2012). The transmission of genes and culture: A questionable analogy. Evolutionary Biology, 39, 12-24.

Danchin, É., et al (2011). Beyond DNA: Integrating inclusive inheritance into an extended theory of evolution. Nature Reviews Genetics, 12, 475-486.

Dobzhansky, T. G. (1956). The Biological Basis of Human Freedom. New York: Columbia University.

Durham, W. H. (1991). Coevolution: Genes, Culture and Human Diversity. Stanford, CA: Stanford University Press.

Feldman, M. W., \& Cavalli-Sforza, L. L. (1989). On the theory of evolution under genetic and cultural transmission with application to the lactose absorption problem. In Mathematical Evolutionary Theory, 145-173. Princeton, NJ: Princeton University Press.

Gerbault, P. et al (2011). Evolution of lactase persistence: An example of human niche construction. Philosophical Transactions of the Royal Society B: Biological Sciences $366,1566,863-77$.

Griffiths, P. E., \& Gray, R. D. (1994). Developmental systems and evolutionary explanation. The Journal of Philosophy, 91, 277-304.

Griffiths, P.E., \& Gray, R. D. (2001) Darwinism and developmental systems. In S. Oyama (Ed), Cycles of Contingency: Developmental Systems and Evolution, 195-218. Cambridge, MA: MIT Press.

Griffiths, P. E., \& Gray, R. D. (2004). The developmental systems perspective: Organism-environment systems as units of evolution. In M. Pigliucci \& K. Preston (Eds.), Phenotypic Integration: Studying the Ecology and Evolution of Complex Phenotypes, 409-431. Oxford: Oxford University Press.

Heyes, C. (2018). Cognitive Gadgets: The Cultural Evolution of Thinking. Harvard University Press. 
Jablonka, E., \& Lamb, M. J. (2005). Evolution in Four Dimensions: Genetic, Epigenetic, Behavioral and Symbolic Variation in the History of Life. Cambridge, MA: MIT Press.

Kroeber, A. L. (1917). The superorganic. American Anthropologist, 19, 163-213.

Kronfeldner, M. (2007). Is cultural evolution Lamarckian? Biology and Philosophy 22, 4, 493-512.

Kronfeldner, M. (2009). If there is nothing beyond the organic... : Heredity and culture at the boundaries of anthropology in the work of Alfred L. Kroeber. NTM Journal of the History of Science, Technology and Medicine, 17, 107-133.

Kronfeldner, M. (2017). The right to ignore: An epistemic defense of the nature/culture divide." In R. Joyce (Ed). Routledge Handbook of Evolution and Philosophy, 210-24. New York: Routledge.

Kronfeldner, M. (2018). What's Left of Human Nature? A Post-Essentialist, Pluralist, and Interactive Account of a Contested Concept. Cambridge, MA: MIT Press.

Lamm, E. (2018). Inheritance systems. In E. N: Zalta (Ed.) The Stanford Encyclopedia of Philosophy (revised version, Summer 2020 Edition), https:// plato.stanford.edu/archives/sum2020/entries/inheritance-systems/.

Lewens, T. (2015). Cultural Evolution: Conceptual Challenges. Oxford: Oxford University Press.

Lewens, T. (2018). Cultural evolution. In E. N. Zalta (Ed.). The Stanford Encyclopedia of Philosophy, Summer 2020. Metaphysics Research Lab, Stanford University. https://plato.stanford.edu/archives/sum2020/entries/evolution-cultural/.

Meloni, M. (2016). From boundary-work to boundary object: How biology left and reentered the social sciences. The Sociological Review Monographs, 64, 1, 61-78.

Merlin, F. (2010). On Griffiths and Gray's concept of expanded and diffused inheritance. Biological Theory, 5, 206-215.

Merlin, F. (2017). Limited extended inheritance. In P. Huneman \& D. Walsh (Eds.), Challenging the Modern Synthesis: Adaptation, Development and Inheritance, 263-79. Oxford: Oxford University Press.

Morin, O. (2015). How Traditions Live and Die. Oxford: Oxford University Press.

Odling-Smee, J. (2007). Niche inheritance: A possible basis for classifying multiple inheritance systems in Evolution. Biological Theory, 2, 276-289.

Odling-Smee, J. (2010). Niche inheritance. In M. Pigliucci \& G. Müller (Eds.), Evolution: The Extended Synthesis, 175-207. Cambridge, MA: MIT Press.

Odling-Smee, J., \& Laland, K. (2011). Ecological inheritance and cultural inheritance: What are they and how do they differ? Biological Theory, 6, 220-30.

Pellett, P. E., et al (2012) Chromosomally integrated human Herpesvirus 6: Questions and answers. Reviews In Medical Virology, 22, 144-155.

Richerson, P. J., \& R. Boyd. (2005). Not by Genes Alone: How Culture Transformed Human Evolution. Chicago: University of Chicago Press.

Simon, H. A. (1962). The architecture of complexity. Proceedings of the American Philosophical Society, 106, 467-482. 
Sperber, D. (1996). Explaining Culture: A Naturalist Approach. Oxford: Blackwell.

Tooby, J., and L. Cosmides. (1992). “The Psychological Foundations of Culture.” In J.

H. Barkow et al (Eds). The Adapted Mind: Evolutionary Psychology and the Generation of Culture, 19-136. Oxford: Oxford University Press. 